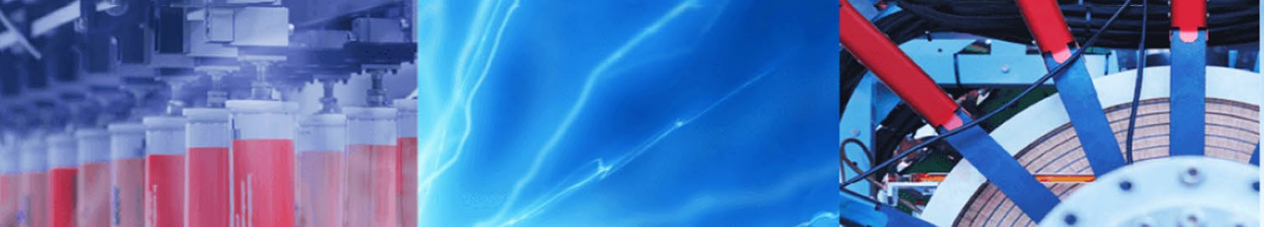

Research Article

\title{
Application of multi-criteria decision-making (MCDM) methods on a meso-scale electrical discharge drilling (EDD) process
}

\author{
Yakup Yildiz ${ }^{1}$ (D)
}

Received: 2 May 2020 / Accepted: 12 October 2020 / Published online: 23 October 2020

(c) Springer Nature Switzerland AG 2020

\begin{abstract}
Multi/Multiple-criteria decision-making methods or multiple-criteria decision analysis (MCDA) have a useful contribution to determine the optimal or the best experimental condition for a process. One of the objectives of the presented research is the observation of the effects of the electrical parameters on the material removal rate (MRR) and the electrode wear ratio (EWR) in a meso-scale EDD. The examined electrical parameters are pulse-on/off time, duty cycle/factor and capacitance. Machining results have been investigated by analysis of variance and graphically depending on their main effects. While pulse-on time and duty cycle have statistically significant effect on MRR, pulse-on time, capacitance and duty cycle have significant effect on EWR for the EDD processes. VIKOR, TOPSIS (Technique for Order Preference by Similarity to Ideal Solution) and GRA (Grey Relation Analysis) were used as MCDM methods to determine the optimum machining result in terms of maximum MRR and minimum EWR. MCDM results have been monitored by graphically. Since an inconsistency has been seen between the MCDM results, a second supplementary analysis has been done by Weighted of Sum and Weighted of Product methods. By this way, a better consistency has been provided. Consequentially, it has been seen that the results of the second modifier analysis and the results of VIKOR were closer to each other in terms of specifying the optimal condition for the process. Hole formations were also observed by scanning electron microscopy images in this work. As a result, it has been decided that the minimum pulse-on time and pulse off-time with $50 \%$ duty cycle and minimum capacitance inputs gives an optimum output for MRR and EWR in the meso-scale EDD process.
\end{abstract}

Keywords MCDM · Meso-scale EDD · Capacitance $\cdot$ Duty cycle $\cdot$ VIKOR $\cdot$ TOPSIS · GRA

\section{Introduction}

Most of the machining operations, like in this study, are not qualified by its single performance measure. Material removal rate and electrode wear ratio are the multiple evaluation criteria of this study. However, reality of their expected opposite dependence makes complication in deciding to the best machining performance. For this reason, EDD performance of this study were analyzed by different multi-criteria decision-making methods [1] to identify the best or the optimal machining condition. These procedures are VIKOR (vlsekriterijumska optimizacija i kompromisno resenje), TOPSIS and GRA. These methods are widely used in manufacturing fields $[2,3]$.

Electro discharge machining (EDM) is a nontraditional manufacturing (NTM) method using thermoelectrically energy for manufacturing geometrically complex and hard material parts that are extremely difficult-to-machine by conventional or traditional machining processes. EDM is among the earliest of NTM processes having had Electronic supplementary material The online version of this article (https://doi.org/10.1007/s42452-020-03668-0) contains
supplementary material, which is available to authorized users.

Yakup Yildiz, yakup.yildiz@live.com | 'Department of Mechanical Engineering, Simav Technology Faculty, Kutahya Dumlupinar University, Kutahya, Turkey. 
its inception 1940s in a simple die-sinking application. Although it is currently being possible to apply EDM in the cases of some insulating materials, electrically conductive materials can be machined by EDM easily. EDM is a non-contact machining technique and the non-contact machining techniques have been attracting a significant amount of industrial and research interest especially for micro-meso scale machining applications [4].

Sometimes referred to as spark machining or spark eroding, EDM is a thermo-electric process that machines the materials from electrical conductive workpieces by an array of intermittent or discontinuous and rapidly recurring electric sparks between tool and workpiece.

In working principle of this process, an electric voltage is applied to the electrodes, and this generates the electric and electromagnetic field. Electric resistance of this field decreases by polarization. This initiates circulation of electrons and ions between the electrodes. Amperage increases and spark occurs. The spark is at plasma temperature and surrounded by a gas sheath. High plasma temperatures causes vaporization of the workpieces. Due to the increase of the material temperature, the development of a melting process and a little later vaporization begins. The most prominent advantages of EDM include machining of complex shapes and extremely hard materials that difficult to machine with conventional methods. However, the inability to machine electrically non-conductive materials, slow rate of materials removal and excessive electrode wear are the most notorious disadvantage of this method [5].

Various researches have been done in number of ways to improve the material removal rate and electrode wear performance of EDM. Though there are lots of approaches, the objective remained the same in terms of achieving more efficient material removal coupled with a reduction in tool wear. This study aimed to analyze meso-scale EDD performance taking into account the electrical parameters such as pulse-on/off time, duty cycle and capacitance. EDD is one of the two principal types of EDM sinking and a process, widely used in the industry to generate holes in very hard and electrically conductive materials in high speed [6].

Numerous studies have been done on EDM/EDD to investigate the effects of electrical parameters on the results. Yu et al. [7] made a comprehensive study on the effect of various parameters such as open circuit voltage, capacitance, electrode feed and layer depth on the material removal rate and the electrode wear ratio. They found that the MRR and EWR increases with increase in capacitance. Kuppan et al. [8] conducted an experiment in EDM of small deep hole drilling of Inconel 718 to study the effect of input parameters like the peak current, pulseon time, duty factor and electrode speed on MRR. By the experiments, they found that the MRR is more influenced by the peak current, duty factor and electrode rotation. Ko-Ta Chiang [9] conducted experiments on the effect of machining parameters on the performance of EDM process. He chose four machining parameters (the discharge current, pulse-on time, duty factor and open discharge voltage) to study the performance characteristics of MRR and EWR. Through his study, while he found out that the discharge current and duty factor are the main influencing parameters on the value of MRR, EWR is more influenced by the discharge current and pulse-on time. Jahan et al. [10] investigated different kinds of electrode performances in micro-EDM. Mahardika et al. [11] worked on micro-EDM performance. Kachab et al. [12] investigated performance evaluation of different electrode geometries in EDD.

Nevertheless, tool wear is inevitable outcome of EDM and EDD processes due to their thermoelectrically mechanism. Moreover, it is a proven truth that higher MRR means also higher EWR in this process. Therefore, it is required to apply MCDM methods to qualify the process performance in the case of both machining results, MRR and EWR. Similar studies have been done in literature widely [13]. Kumar and Soota [14] proposed grey relation analysis to optimize EDM process parameters. Payal et al. [15] employed GRA to optimize the process input parameters during EDM of an Inconel material. Pradhan [16] suggested GRA and TOPSIS to assess process parameters of EDM. Bhuyan et al. [17] optimized process parameters by VIKOR for EDM process of a metal matrix composite. Pattnaik et al. [18] used fuzzy TOPSIS in order to optimize EDM parameters for a stainless steel. Guo et al. [19] used gray relation analysis method for another EDM drilling process. They selected peak current, pulse duration, duty factor and flushing pressure as the machining parameters. Machining time, electrode wear and surface roughness were chosen to evaluate the effects of machining. Manivannan and Kumar [20] used TOPSIS method for multi attribute decision making of micro-EDM drilling process parameters. The input parameters were current, pulse on/off durations and gap voltage. The observed outputs were geometrical characterizations, material removal rate, tool wear rate and average roughness. Bhosle and Sharma [21] applied GRA to investigate multi-performance optimization of micro-EDM drilling process of Inconel 600 alloy. They varied five process parameters such as voltage, capacitance, EDM federate, pulse-on time and pulse-off time in drilling process using tungsten carbide tool. They determined optimum material removal rate and minimum taper, overcut and diametric variation by this way. Sapkal and Jagtap [22] examined optimization of micro EDM drilling process parameters for titanium alloy by copper tungsten electrode. They used response surface methodology based central composite design. They analyzed the process parameters like pulse 
on time, discharge voltage, capacitance and electrode rotation speed depending on material removal rate, side gap width and taper ratio.

In this study, firstly, experimental results have been analyzed by analysis of variance (ANOVA) and graphically. However, due to nonlinear of some results, secondly, multi-criteria decision-making (MCDM) methods, VIKOR, TOPSIS and GRA were employed to evaluate and compare the machining results of EDD in terms of their MRR and EWR results for the most appropriate options. Since these first group of examination was unsatisfying for the aim, a second group of screening was also practiced by weighted sum (WS) and weighted product (WP) methods as a third stage. Hence, a satisfactory result was obtained and it was decided to optimal machining condition of EDD process in this study. So, the aim of this study is not only to determine optimal machining conditions of an EDD process by MCDM methods, but also to make a comparison between these methods and to normalize their result to achieve the most reliable result. SEM images of the created holes are also given a separate section in the study. Flow diagram of the whole study is given in Fig. 1 .

\section{Experimental procedure}

\subsection{Machine and electrical parameters}

Experiments were conducted on a ZNC/50A electro discharge drilling machine (hole popper) produced by Corisma Inc. in Taiwan. The effect of the input parameters such as pulse-on/off time, duty cycle and capacitance were examined in terms of their effects on material removal rate and electrode wear.
Pulse-on time is the amount of current or discharge that flow into the gap between the two electrodes. Pulse of time is the amount of interval, the power and so discharge is interrupted during the EDM cycle. Capacitance is the measure of amount of electric charge stored for a given electric potential. Duty cycle is an indicator of the relationship between on-time and off-time and the efficiency of the process. It is determined by the Eq. 1,

$D C=\frac{t_{\text {on }}}{t_{\text {on }}+t_{\text {off }}} * 100$

where $D C$ is the duty cycle (\%), $t_{\text {on }}$ is pulse-on time ( $\left.\mu \mathrm{s}\right)$ and $t_{\text {off }}$ is pulse-on time ( $\left.\mu \mathrm{s}\right)$.

\subsection{Workpiece, tool and dielectric medium}

Workpiece specimen was Protherm 17500 Copper-Beryllium (Cu-Be) Alloy with $26 \mathrm{~mm} \times 26 \mathrm{~mm} \times 13 \mathrm{~mm}$ in dimensions. Chemical composition of the workpiece includes $0.6 \% \mathrm{Be}$ and $2.6 \% \mathrm{Co}$ and the rest Cu by weight. Lower beryllium content of Protherm material differs from Moldmax material. Density of the workpiece is $5.109 \mathrm{~kg} / \mathrm{m}^{3}$, elastic modulus is $20 \times 10^{6} \mathrm{psi}$, thermal expansion coefficient $9.8 \times 10^{-6}$ in./in. $/{ }^{\circ} \mathrm{F}, 70-400^{\circ} \mathrm{F}$. Thermal conductivity of the material is $115 \mathrm{Btu} /\left(\mathrm{ft} . \mathrm{hr} .{ }^{\circ} \mathrm{F}\right)$. Melting point of the material is $1850-1930^{\circ} \mathrm{F}$ [23]. Workpiece was placed properly on a special fixture mounted on the magnetic bed.

Electrode tool was single hole copper tube with $\varnothing 1$ $\mathrm{mm}$ outer diameter, $\varnothing 0.3 \mathrm{~mm}$ inner diameter and $400 \mathrm{~mm}$ length in dimensions. Chemical composition of the copper electrode includes $99.9 \% \mathrm{Cu}$ and $0.015-0.04$ Phosphorus (P) by weight. Density of the electrode is $8.94 \mathrm{~g} / \mathrm{mm}^{3}$.

Fig. 1 Flow diagram of the study

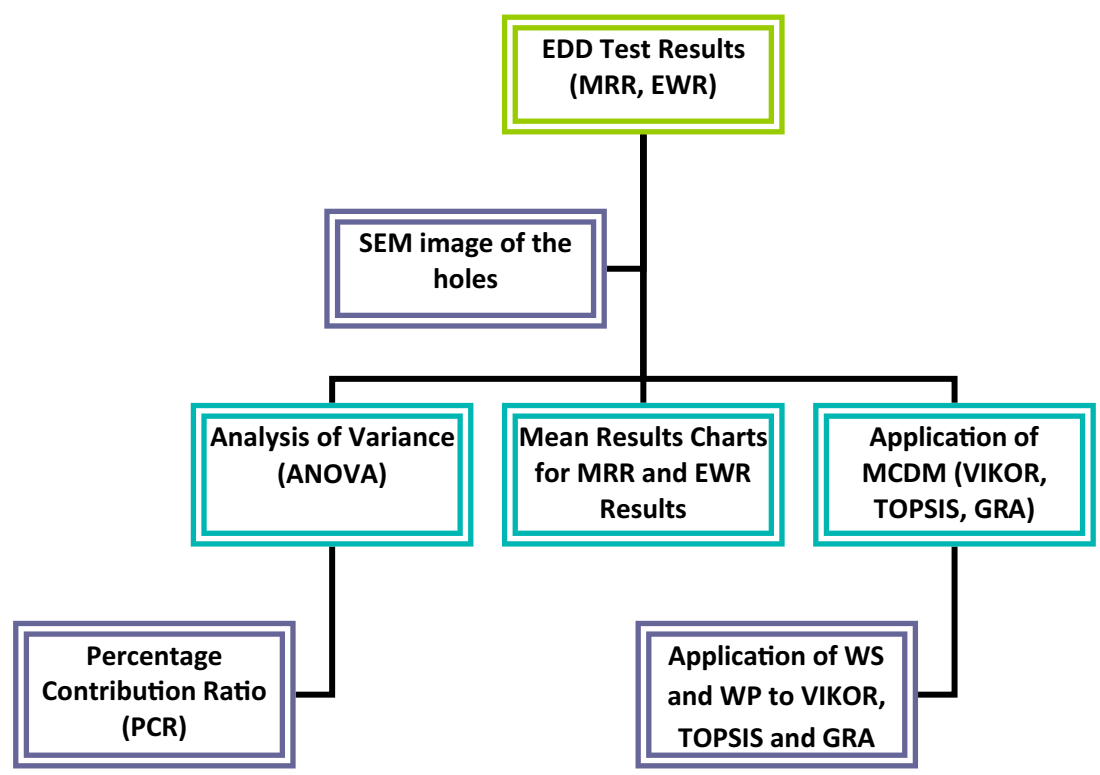


Deionized water was used as dielectric medium. Some properties of the medium are, density is $8.34 \mathrm{lbs} / \mathrm{Gal}$, dielectric strength is $13 \mathrm{MV} / \mathrm{m}$, dynamic viscosity is $0.92 \mathrm{~g} /$ $\mathrm{ms}$, thermal conductivity is $0.606 \mathrm{~W} / \mathrm{mK}$ and specific heat capacity is $4.19 \mathrm{~J} / \mathrm{gK}$. Both side and inside flushing method was used in the experiments. Dielectric nozzle position for the side flushing was adjusted so that it would properly flush the debris or removed material. Dielectric flushing pressure was set as $25 \mathrm{~kg} / \mathrm{cm}^{2}$ under whole condition.

\subsection{Machining tests and outputs}

Figure 2 depicts the photograph of the machine and materials used for the experimentation. For the electrode, a proper distance was set between the tool holder and tool guide so that oscillation of the tool was minimized while rotating. An appropriate distance between the electrode and workpiece was also set $(\sim 2.6 \mathrm{~mm})$ automatically from the control unit of the machine. Depth of $10 \mathrm{~mm}$ was set for the Z-axis to drill the hole.

The material removal rates (MRR) and the total electrode tool wear ration (EWR) were measured for each set of parameters from the weight difference concepts of the parts both before and after the EDD process using a sensitive micro-level balance (Sartorius precision scale type E-1200S) having $1210 \mathrm{~g}$ capacity and $0.001 \mathrm{~g}$ resolution. MRR was calculated by the Eq. 2,
$\operatorname{MRR}=\frac{\left[W_{w b}-W_{w a}\right]}{\rho * t_{\mathrm{sec}}}$

where $\mathrm{W}_{\mathrm{wb}}$ is the weight of the workpiece before EDD, $\mathrm{W}_{\text {wa }}$ is the weight of the workpiece after EDD, $\rho$ is the density of the used workpiece material and $t_{s}$ is the time of the EDD process. On the other hand, EWR was calculated by the following Eq. 3,

$E W R=\frac{V_{e t}}{V_{w}} * 100$

where $V_{\text {et }}$ is the volume difference of the electrode tool and $V_{w}$ is the volume difference of the workpiece before and after EDD process for both materials.

Table 1 summarizes the EDD parameter settings adopted for the present study. It can be seen that the capacitance ranges from 1 to 11 , the pulse durations are 25-75 and duty cycles are 25-75. Hence, the totally 27 main experiments with one repetition were adapted for the study based on randomized full factorial design $\left(3^{3}\right)$. The other parameters such as working voltage, working current, GAP voltage, spindle speed, servo feed speed were fixed as $150 \mathrm{~V}, 25 \mathrm{~A}, 16 \mathrm{~V}, 6 \mathrm{rpm}$ and $50 \mathrm{~mm} / \mathrm{min}$ respectively according to the supplier recommendations.

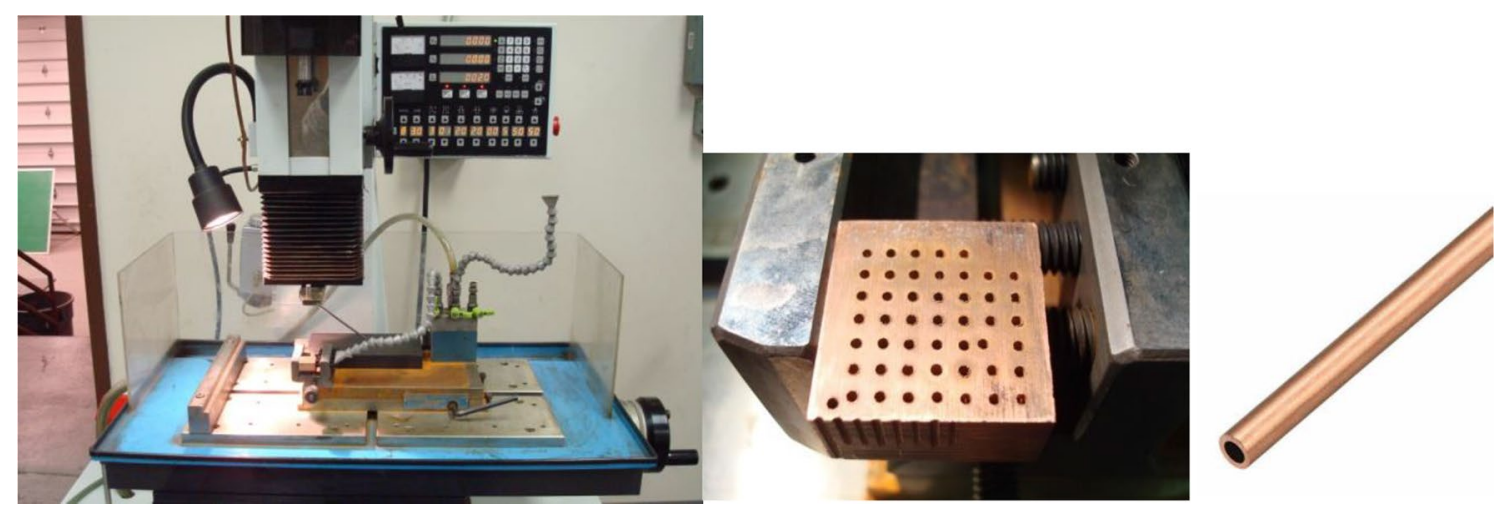

Fig. 2 Electro Discharge Drilling (EDD) machine (hole popper), Protherm C17500 copper-beryllium alloy specimen machined with EDD and a sample copper tube electrode

Table 1 Design of experiment (DOE)

\begin{tabular}{llllllll}
\hline EDM inputs & Level 1 & Level 2 & Level 3 & Level 4 & Level 5 & Level 6 & Level 7 \\
\hline Pulse-on duration $(\mu \mathrm{s})$ & 25 & 50 & 75 & - & - & - & - \\
Pulse-off duration $(\mu \mathrm{s})$ & 25 & 50 & 75 & - & - & - & - \\
Capacitance $(\mu \mathrm{F})$ & 1 & 6 & 11 & - & - & - & - \\
Duty factor $(\%)$ & 25 & 33 & 40 & 50 & 60 & 67 & 75 \\
\hline
\end{tabular}




\section{Analytical procedure}

\subsection{Analysis of variance (ANOVA)}

Univariate Analysis of Variance (ANOVA) for the dependent variables material removal rate (MRR) and electrode wear ratio (EWR) was used for the statistical analysis of the numerical results to determine the significance of the independent electrical factors. ANOVA was supported by PCR (Percentage Contribution Ratio) to specify contributions of each factor and interactions between them. Equation 4 gives $\mathrm{PCR}$,

$P C R_{f i}=\left[\left(\frac{S S_{f i}-\left[M S_{e} * d f_{f i}\right]}{S S_{C T}}\right)\right] * 100$

where $P C R_{f}$ is the percentage contribution of the related factor, $\mathrm{SS}_{\mathrm{f}}$ is the sum of squares of each factor in the source column, $\mathrm{MS}_{\mathrm{e}}$ is the error of mean square, $\mathrm{df}_{\mathrm{f}}$ is the degree of freedom of each factor and $\mathrm{SS}_{\mathrm{CT}}$ is the corrected total of the sum of squares.

\subsection{Performance measures with VIKOR}

This technique, which is depending on the particular measure of "closeness" to the "ideal" solution, was developed by Opricovic [24]. The ranking procedure of this technique can be determined by the following steps [25].

1. Determination of weightage of the machining results.

Table 6 shows the numerical solutions that MRR is the beneficial criterion and EWR is the unbeneficial criterion.

2. Finding best and worst values for each criterion.

Best is $\left(X_{i j}\right)_{\max }$ for beneficial and $\left(X_{i j}\right)_{\min }$ for unbeneficial criterion. Worst is $\left(X_{i j}\right)_{\min }$ for beneficial and $\left(X_{i j}\right)_{\max }$ for unbeneficial criterion.

3. Calculation of $S_{i}$, uniquely measure by the Eq. 5,

$$
S_{i}^{M R R, E W R}=\left[\sum_{j=1}^{n}\left(W_{j} * \frac{X_{i}^{+}-X_{i j}}{X_{i}^{+}-X_{i}^{-}}\right)^{P}\right]^{\frac{1}{p}}
$$

where $\mathrm{n}$ is the number of the alternatives, $\mathrm{X}_{\mathrm{i}}^{+}$is the best alternatives of MRR and EWR, $X_{i}^{-}$is the worst alternatives of MRR and EWR. $X_{i j}$ is each alternative of $M R R$ and EWR. If $p=1$ then all criterion deviations are weighted equally. If $p=2$ then the criterion deviations are weighted based on their magnitudes.

4. Calculation of total $\mathrm{S}_{\mathrm{i}}$ by the Eq. 6
$S_{i}=S_{i}^{M R R}+S_{i}^{E W R}$

5. Determination of $R_{i}$, which is maximum value of $S_{i}$ MRR and $S_{i}^{E W R}$ row.

6. Finding out $S^{\#}, S^{-}, R^{\#}$ and $R^{-}$values by the following equations,

$S^{\#}=\min S_{i-} S^{-}=\max S_{i-} R^{\#}=\min R_{i-} R^{-}=\max R_{i}$

7. Calculation of $Q_{i}$ by the Eq. 8,

$Q_{i}=v * \frac{S_{i}-S^{\#}}{S^{-}-S^{\#}}+(1+v) * \frac{R_{i}-R^{\#}}{R^{-}-R^{\#}}$

where $v$ is the weight for strategy of maximum group utility, which is taken as 0.5 in the analysis. Because generally, it takes the value 0.5 [25].

8. Determination of the ranking based on $Q_{i}$ values.

The minimum value of $Q_{i}$ will be the best solution. However, the following two conditions must be satisfied simultaneously.

C1 (Acceptable advantage): $Q\left(A^{2}\right)-\left(A^{1}\right) \geq D Q$, where $A^{1}$ and $A^{2}$ are the alternatives with the first and second highest rank in the ranking list respectively and DQ is $\frac{1}{j-1}$, where $\mathrm{j}$ is number of the alternatives.

C2 (Acceptable stability in decision making): $A^{1}$ should also be the best ranked by $\mathrm{S}_{\mathrm{i}}$ or/and $\mathrm{R}_{\mathrm{i}}$.

Unfortunately, these two conditions mostly cannot be achieved simultaneously. Then a set of following compromise solutions is proposed.

S1 If condition $C 1$ is not satisfied, $A^{n}$ in alternatives $A^{1}, A^{2}, \ldots, A^{n}$ is determined by the relation $Q\left(a^{n}\right)-Q\left(a^{1}\right)<D Q$ for maximum $\mathrm{n}$ (the positions of these alternatives are "in closeness").

S2 If only condition C2 is not satisfied, the alternatives $A^{1}$ and $A^{2}$ are the compromise solutions.

\subsection{Performance measures with TOPSIS}

The ranking procedure of this method can be determined by the following steps [26].

1. Determination of weightage of the machining results. MRR is the beneficial criterion and EWR is the unbeneficial criterion. Their weightages are equal as 0.5 as in VIKOR technique.

2. Calculation of Normalized Matrix by Eq. 9 ,

$$
\bar{X}_{i j}=\frac{X_{i j}}{\sqrt{\sum_{j=1}^{n} X_{i j}^{2}}}
$$


where $X_{i j}$ each value of MRR and EWR results.

3. Calculation of weighted Normalized Matrix by Eq. 10,

$$
V_{i j}=\bar{X}_{i j} * W_{j}
$$

4. Determination of ideal best and ideal worst values of $\mathrm{V}_{\mathrm{ij}}$

5. Calculation of Euclidean distance from the ideal best and worst by Eq. 11,

$S_{i}^{ \pm}=\left[\sum_{j=1}^{n}\left(V_{i j}-V_{j}^{ \pm}\right)^{2}\right]^{0.5}$

6. Calculation of Performance Score by Eq. 12,

$$
P_{i}=\frac{S_{i}^{-}}{S_{i}^{+}+S_{i}^{-}}
$$

7. Determination of the best ranking value depending on the biggest performance score value

\subsection{Performance measures with GRA}

The ranking procedure of this method can be determined by the following steps [27].

1. The experimental results of the MRR and EWR have been normalized in the range between 0 and 1 by grey relational generating. The best normalized result will be equal to 1 , which express the better performance. The normalized experimental values of the $\operatorname{MRR}\left(Y_{i}(n)\right)$ were calculated by Eq. 13 with regard to higher-thebetter performance characteristic,

$Y_{i}(n)=\frac{X_{i}(n)-\min X_{i}(n)}{\max X_{i}(n)-\min X_{i}(n)}$

where $\mathrm{Xi}(\mathrm{n})$ is the $\mathrm{nth}$ experimental result in the ith experiment, $\min _{i}(n)$ and $\max _{i}(n)$ are the minimum and maximum values of $X_{i}(n)$ respectively. And the normalized experimental results $Y_{i}(n)$ of EWR can be calculated by Eq. 14 with regard to lower-the-better performance characteristic,

$Y_{i}(n)=\frac{\max X_{i}(n)-X_{i}(n)}{\max X_{i}(n)-\min X_{i}(n)}$

2. The grey relational coefficients expressing the relation between the best and actual normalized values are calculated by the following Eqs. 15 and 16,

$\Delta_{0 i}(n)=\left\|Y_{0}(n)-Y_{i}(n)\right\|$

$$
\xi_{i}(n)=\frac{\Delta_{\min }+\zeta * \Delta_{\max }}{\Delta_{0 i}(n)+\zeta * \Delta_{\max }}
$$

where $\Delta_{0 \mathrm{i}}(\mathrm{n})$ is the absolute differences of two comparative sequence, $Y_{0}(n)$ is the reference sequence which is equal to 1 and $Y_{i}(n)$ is the comparison sequence in the analysis, $\xi_{i}(n)$ is the grey relational coefficient, $\Delta_{\min }$ and $\Delta_{\max }$ is the minimum and the maximum values of $\Delta_{0 i}(\mathrm{n})$ respectively, $\zeta$ is the weight coefficient defined as 0.5 as in the other methods. $\xi_{i}(\mathrm{k})$ is the grey relational coefficient and the highest value of $\xi_{i}(k)$ represents that the related experimental result is closer to the best normalized value for the single performance characteristic.

3. The grey relational grade is calculated by the Eq. 17 for evaluation of the multi process characteristics by finding the mean value of the grey relational coefficients,

$\gamma_{i}=\frac{\sum_{k=1}^{m} \xi_{i}(m)}{m}$

where $\gamma_{\mathrm{i}}$ is the grey relational grade and $\mathrm{m}$ is the number of performance characteristics. The higher grey relational grade refers the higher rank for the multiple performance characteristic.

\section{Results and discussion}

Experimental results have been discussed based on the main effects of the electrical parameters on MRR and EWR by ANOVA and by graphically. Numerical results of the experiments made it compulsory to carry out an optimization study. Therefore, VIKOR, TOPSIS and GRA methods were used to determine optimum result in terms of MRR and EWR. SEM images of the meso-scale drilled holes have been also shown in a separate section.

\subsection{The effects of the electrical parameters on MRR}

Tables 2 and 3 shows univariate analysis of variance (ANOVA) results. The analysis of variance including the two levels interaction shows better fitting results for full factorial design of experiments. Therefore, a second analysis was implemented to determine the significance of duty cycle and capacitance. Thus, the only parameters that have significant effect on the material removal rate is the pulse-on time and duty cycle based on 0.05 significance level and 95\% confidence interval. Their PCR results on the variation are $24.1 \%$ and $63.5 \%$ respectively. This result of duty cycle is coherent with results of Kuppan et al. [8] and Ko-Ta Chiang [9]. The capacitance and the pulse-on time have no main effect on the material removal rate. The 
Table 2 Univariate analysis of variance (ANOVA) for dependent variable MRR based on two levels interactions of pulse-on time, pulse-off time and capacitance

\begin{tabular}{lccccc}
\hline Source & $\begin{array}{l}\text { Type III sum of } \\
\text { squares }\end{array}$ & df & Mean square & F & Sig \\
\hline Pulse_On_Time & 56,484 & 2 & 28,242 & 13,011 & .003 \\
Pulse_Off_Time & 11,422 & 2 & 5711 & 2631 & .132 \\
Capacitance & 10,660 & 2 & 5330 & 2456 & .147 \\
Pulse_Off_Time*Capacitance & 16,988 & 4 & 4247 & 1957 & .194 \\
Pulse_On_Time*Capacitance & 12,988 & 4 & 3247 & 1496 & .290 \\
Pulse_On_Time*Pulse_Off_Time & 90,008 & 4 & 22,502 & 10,367 & .003 \\
Error & 17,365 & 8 & 2171 & & \\
Total & 1277,281 & 27 & & & \\
Corrected total & 215,914 & 26 & & & \\
R Squared $=, 920$ & & & & &
\end{tabular}

Table 3 Univariate analysis of variance (ANOVA) for dependent variable MRR based on two levels interactions of capacitance and duty cycle

\begin{tabular}{|c|c|c|c|c|c|}
\hline Source & $\begin{array}{l}\text { Type III } \\
\text { sum of } \\
\text { squares }\end{array}$ & df & Mean square & $\mathrm{F}$ & Sig \\
\hline Capacitance & 9528 & 2 & 4764 & 1586 & .280 \\
\hline Duty cycle & 155,191 & 6 & 25,865 & 8610 & .010 \\
\hline $\begin{array}{l}\text { Capacitance }{ }^{*} \text { Duty } \\
\text { cycle }\end{array}$ & 32,038 & 12 & 2670 & .889 & .596 \\
\hline Error & 18,025 & 6 & 3004 & & \\
\hline Total & 1277,281 & 27 & & & \\
\hline $\begin{array}{l}\text { Corrected total } \\
\text { R Squared }=, 917\end{array}$ & 215,914 & 26 & & & \\
\hline
\end{tabular}

interactions between the pulse-on time and the pulse-off time has also a significant effect on the material removal rate with $37.7 \%$ PCR. However other interactions between the other parameters have no significant effects on MRR.

Figure 3 shows the interactive graphs of the main effects of the electrical parameters, pulse-on/off times, duty cycle and capacitance, on MRR respectively. That means, for example, MRR formation at $25 \mu \mathrm{s}$ is obtained at mean values of three different pulse-off times $(25,50$ and $75 \mu \mathrm{s})$, three different capacitances (1, 6 and $11 \mu \mathrm{F})$ and three different duty cycle $(25,33$ and 50$)$. Based on the mean results, MRR was $5.31 \mathrm{~mm}^{3} / \mathrm{min}$ at $25 \mu$ s pulse-on time. MRR increased to $8.31 \mathrm{~mm}^{3} / \mathrm{min}$ at $50 \mu$ s pulse-on time with $56.5 \%$ variation. However, it decreased again to $5.18 \mathrm{~mm}^{3} / \mathrm{min}$ at $75 \mu$ s pulse-on time with $37.7 \%$ variation. Theoretically the higher pulse-on time means the larger the craters or pits and the faster cut. Therefore, it is expected an increase with increasing pulse-on time. Because, the influence time of the total energy to the workpiece by working current extends by increasing of pulse-on time. In longer pulse durations, the plasma has enough time to increase its radius with an increase in pulse duration, as determined in lots of study [28]. However, the short pulses cause less vaporization from the workpiece and long pulses cause the plasma channel to expand. The expansion of the plasma channel lead to reduction of spark energy density (i.e. energy per unit volume). The supply of low spark energy will reduce the heat input for crater volume, which means insufficient to melt and vaporize the workpiece material [8]. Therefore, material removal rate will decrease after reaching maximal effort like in this study. Similar trends were put forward by other researchers [12, 29-32].

On the other hand, MRR was $6.72 \mathrm{~mm}^{3} / \mathrm{min}$ at $25 \mu \mathrm{s}$ pulse-off time. MRR decreased to $5.35 \mathrm{~mm}^{3} / \mathrm{min}$ at $50 \mu \mathrm{s}$ pulse-off time with $20.4 \%$ variation. However, it increased again to $6.74 \mathrm{~mm}^{3} / \mathrm{min}$ at $75 \mu$ s pulse-off time with $25.9 \%$ variation. This tendency seems to be a mirrored condition of pulse-on time graph. Similar trends were also seen in a recent research [12]. The decrease in MRR with increase pulse-off time is an expected situation due to longer machining times. Intensity of discharge sparking also decreases with longer pulse-off times. However, increase in MRR with longer off-time could be explained by flushing efficiency and so removing debris from the machining zone effectively. The variation of the effect of duty cycle on MRR seems to be like a harmonic motion in the graph. The variation is not linear and therefore the effect of duty cycle on MRR seems not stable. MRR was $5.72 \mathrm{~mm}^{3} / \mathrm{min}$ at $1 \mu \mathrm{F}$ capacitance. MRR increased to $5.94 \mathrm{~mm}^{3} / \mathrm{min}$ at $6 \mu \mathrm{F}$ capacitance with $3.8 \%$ variation. It continued to increase to $7.15 \mathrm{~mm}^{3} / \mathrm{min}$ at $11 \mu \mathrm{F}$ capacitance with $20.4 \%$ variation. Increase of capacitance increases discharge energy. This result of the effect of capacitance on MRR is coherent with results of Yu et al. [7], Jahan et al. [10] and Mahardika et al. [11]. 

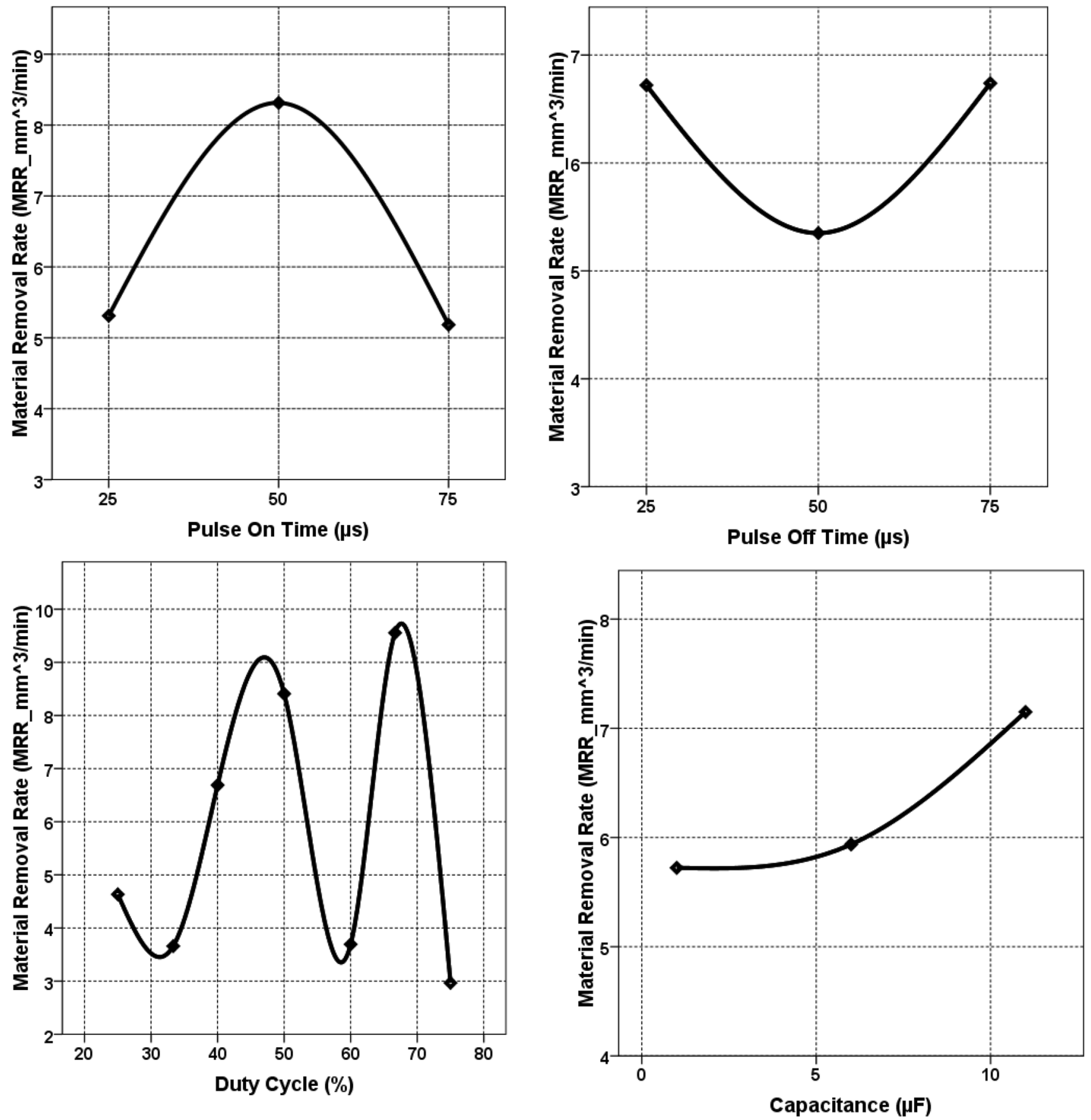

Fig. 3 The effects of the electrical parameters, pulse-on/off times, duty cycle and capacitance, on MRR

\subsection{The effects of the electrical parameters on EWR}

Table 4 and Table 5 shows univariate analysis of variance (ANOVA) results based on 0.05 significance level and 95\% confidence interval. A second analysis was also implemented to determine the significance of duty cycle and capacitance on EWR. The parameters that have effect on EWR are the pulse on-time, capacitance and duty cycle. Their PCR results on the variation are $14.6 \%, 19.9 \%$ and
$51 \%$ respectively. Pulse off-time has no main effect on the EWR. The only interaction that has effect on EWR is between the pulse on-time and pulse off-time with $27.6 \%$ $P C R$. Other interactions are seeming insignificant.

Figure 4 shows the interactive graphs of the main effects of the electrical parameters, pulse-on/off times, duty cycle and capacitance, on EWR respectively. Based on the mean results, total wear was $39.1 \%$ at $25 \mu$ s pulseon time. Total wear decreased to $33.8 \%$ at $50 \mu$ s pulse-on 
Table 4 Univariate analysis of variance (ANOVA) for dependent variable EWR

\begin{tabular}{lcclcc}
\hline Source & $\begin{array}{l}\text { Type III sum of } \\
\text { squares }\end{array}$ & df & Mean square & F & Sig \\
\hline Pulse_On_Time & 7616 & 2 & 3808 & 6648 & .020 \\
Pulse_Off_Time & 2386 & 2 & 1193 & 2083 & .187 \\
Capacitance & 10,019 & 2 & 5009 & 8745 & .010 \\
Pulse_Off_Time*Capacitance & 2799 & 4 & .700 & 1222 & .374 \\
Pulse_On_Time*Capacitance & 2439 & 4 & .610 & 1065 & .433 \\
Pulse_On_Time*Pulse_Off_Time & 14,529 & 4 & 3632 & 6341 & .013 \\
Error & 4583 & 8 & .573 & & \\
Total & 178,707 & 27 & & & \\
Corrected total & 44,370 & 26 & & & \\
R Squared $=, 897$ & & & & & \\
\hline
\end{tabular}

\begin{tabular}{lcrlrl}
\hline Source & $\begin{array}{l}\text { Type III Sum of } \\
\text { squares }\end{array}$ & df & Mean square & F & Sig \\
\hline Capacitance & 8676 & 2 & 4338 & 17,363 & .003 \\
Duty_Cycle & 24,145 & 6 & 4024 & 16,106 & .002 \\
Capacitance*Duty_Cycle & 8708 & 12 & .726 & 2904 & .100 \\
Error & 1499 & 6 & .250 & & \\
Total & 178,707 & 27 & & & \\
Corrected total & 44,370 & 26 & & & \\
R Squared =,966 & & & & & \\
\hline
\end{tabular}

Table 5 Univariate analysis of variance (ANOVA) for dependent variable EWR benefit from the maximum duty factor to get minimum electrode tool wear in EDD operations depending on machine capacity. This means, to operate higher pulse-on time and lower pulse-off time in EDD operations especially during drilling with meso-scale electrode tools, which causes working with lower frequency and higher power combination. Total wear was $27.42 \%$ at $1 \mu \mathrm{F}$ capacitance. It increased to $31.42 \%$ at $6 \mu \mathrm{F}$ capacitance with $14.6 \%$ variation. It continued to increase to $42.65 \%$ at $11 \mu \mathrm{F}$ capacitance with $35.7 \%$ variation. Increase of capacitance increases the discharge energy. This result of the effect of capacitance on EWR is coherent with results of Yu et al. [7], Jahan et al. [10] and Mahardika et al. [11]. Hence it is suggested to work with minimum capacitance to get minimum electrode tool wear in EDD operations.

\subsection{MCDM analyses}

The determined optimal conditions depending on the different MCDM methods have been shown in Fig. 5 by their rankings. According to this graph, the best machining condition to get optimal MRR and EWR performances is achieved by test 1 ( $25 \mu$ s pulse-on/off time $50 \%$ duty factor and $1 \mu \mathrm{F}$ capacitance) depending on the VIKOR. The condition $\mathrm{C} 1$ is not provided in this study by VIKOR, but alternative $A^{1}$ is the best ranked by $R_{i}$, which provides the 
Table 6 Performance score results based on different MCDM methods

\begin{tabular}{|c|c|c|c|c|c|c|c|c|c|c|c|}
\hline \multirow[t]{4}{*}{ Alternatives } & \multicolumn{4}{|c|}{ Experimental machining variables } & \multicolumn{3}{|c|}{$\begin{array}{l}\text { Performance scores based on } \\
\text { different MCDM methods }\end{array}$} & \multicolumn{2}{|c|}{$\begin{array}{l}\text { Performance } \\
\text { scores }\end{array}$} & \multicolumn{2}{|c|}{$\begin{array}{l}\text { Rank } \\
\text { scores }\end{array}$} \\
\hline & \multirow{3}{*}{$\begin{array}{l}\text { Pulse-on } \\
\text { time }(\mu s)\end{array}$} & \multirow{3}{*}{$\begin{array}{l}\text { Pulse-off } \\
\text { time }(\mu \mathrm{s})\end{array}$} & \multirow[t]{3}{*}{ Duty cycle (\%) } & \multirow[t]{3}{*}{ Capac. $(\mu \mathrm{F})$} & VIKOR & TOPSIS & GRA & & & & \\
\hline & & & & & \multicolumn{3}{|c|}{ Weightage values } & \multirow[t]{2}{*}{ WS } & \multirow[t]{2}{*}{ WP } & \multirow[t]{2}{*}{ WS } & \multirow[t]{2}{*}{ WP } \\
\hline & & & & & 0.33 & 0.33 & 0.33 & & & & \\
\hline 1 & 25 & 25 & 50 & 1 & 0.065 & 0.649 & 0.588 & 0.917 & 0.028 & 1 & 1 \\
\hline 2 & 25 & 25 & 50 & 6 & 0.382 & 0.546 & 0.552 & 0.576 & 0.004 & 10 & 10 \\
\hline 3 & 25 & 25 & 50 & 11 & 0.911 & 0.265 & 0.400 & 0.337 & 0.001 & 24 & 24 \\
\hline 4 & 25 & 50 & 33 & 1 & 0.786 & 0.413 & 0.493 & 0.456 & 0.001 & 18 & 19 \\
\hline 5 & 25 & 50 & 33 & 6 & 0.815 & 0.246 & 0.397 & 0.329 & 0.001 & 25 & 25 \\
\hline 6 & 25 & 50 & 33 & 11 & 1.000 & 0.210 & 0.379 & 0.298 & 0.000 & 27 & 27 \\
\hline 7 & 25 & 75 & 25 & 1 & 0.478 & 0.417 & 0.463 & 0.461 & 0.002 & 17 & 16 \\
\hline 8 & 25 & 75 & 25 & 6 & 0.758 & 0.310 & 0.420 & 0.373 & 0.001 & 23 & 23 \\
\hline 9 & 25 & 75 & 25 & 11 & 0.873 & 0.213 & 0.386 & 0.306 & 0.000 & 26 & 26 \\
\hline 10 & 50 & 25 & 67 & 1 & 0.221 & 0.591 & 0.555 & 0.641 & 0.007 & 6 & 4 \\
\hline 11 & 50 & 25 & 67 & 6 & 0.093 & 0.670 & 0.615 & 0.840 & 0.021 & 2 & 2 \\
\hline 12 & 50 & 25 & 67 & 11 & 0.488 & 0.554 & 0.689 & 0.631 & 0.004 & 8 & 8 \\
\hline 13 & 50 & 50 & 50 & 1 & 0.340 & 0.501 & 0.502 & 0.538 & 0.004 & 14 & 13 \\
\hline 14 & 50 & 50 & 50 & 6 & 0.240 & 0.600 & 0.572 & 0.645 & 0.007 & 4 & 5 \\
\hline 15 & 50 & 50 & 50 & 11 & 0.127 & 0.678 & 0.716 & 0.829 & 0.018 & 3 & 3 \\
\hline 16 & 50 & 75 & 40 & 1 & 0.360 & 0.528 & 0.523 & 0.558 & 0.004 & 12 & 11 \\
\hline 17 & 50 & 75 & 40 & 6 & 0.711 & 0.432 & 0.504 & 0.473 & 0.001 & 16 & 17 \\
\hline 18 & 50 & 75 & 40 & 11 & 0.470 & 0.517 & 0.543 & 0.548 & 0.003 & 13 & 14 \\
\hline 19 & 75 & 25 & 75 & 1 & 0.576 & 0.519 & 0.676 & 0.601 & 0.003 & 9 & 12 \\
\hline 20 & 75 & 25 & 75 & 6 & 0.724 & 0.408 & 0.479 & 0.449 & 0.001 & 19 & 18 \\
\hline 21 & 75 & 25 & 75 & 11 & 0.758 & 0.386 & 0.462 & 0.429 & 0.001 & 21 & 21 \\
\hline 22 & 75 & 50 & 60 & 1 & 0.756 & 0.389 & 0.465 & 0.432 & 0.001 & 20 & 20 \\
\hline 23 & 75 & 50 & 60 & 6 & 0.518 & 0.446 & 0.484 & 0.482 & 0.002 & 15 & 15 \\
\hline 24 & 75 & 50 & 60 & 11 & 0.765 & 0.313 & 0.421 & 0.374 & 0.001 & 22 & 22 \\
\hline 25 & 75 & 75 & 50 & 1 & 0.296 & 0.517 & 0.509 & 0.559 & 0.004 & 11 & 9 \\
\hline 26 & 75 & 75 & 50 & 6 & 0.246 & 0.593 & 0.566 & 0.637 & 0.007 & 7 & 6 \\
\hline 27 & 75 & 75 & 50 & 11 & 0.393 & 0.578 & 0.667 & 0.643 & 0.005 & 5 & 7 \\
\hline
\end{tabular}

condition C2. Since $Q\left(A^{3}\right)-Q\left(A^{1}\right)>D Q$, both $A^{1}, A^{2}$ are assumed compromise solutions in terms of VIKOR [33]. On the other hand, the rank of this test condition is 3 and 6 depending on TOPSIS and Grey Relation Analysis respectively. These methods show that the test 15 (50 $\mu$ s pulseon/off time $50 \%$ duty factor and $11 \mu \mathrm{F}$ capacitance) shows the best performance (rank 1) in terms of optimal MRR and EWR performances. However, the rank of this test is 3 in regard of VIKOR technique.
It is obvious that there is an inconsistency between these methods and it is essential a supplemental examination to decide for the optimal performance measure. For this purpose, weighted sum and weighted product models [34] were implemented to performance scores of VIKOR, TOPSIS and GRA methods. These two methods are also used for solving multi-criteria decision-making problems. In these analyses, performance scores of VIKOR technique, whose lower value is desired, was categorized as unbeneficial criterion. Conversely, performance scores of 

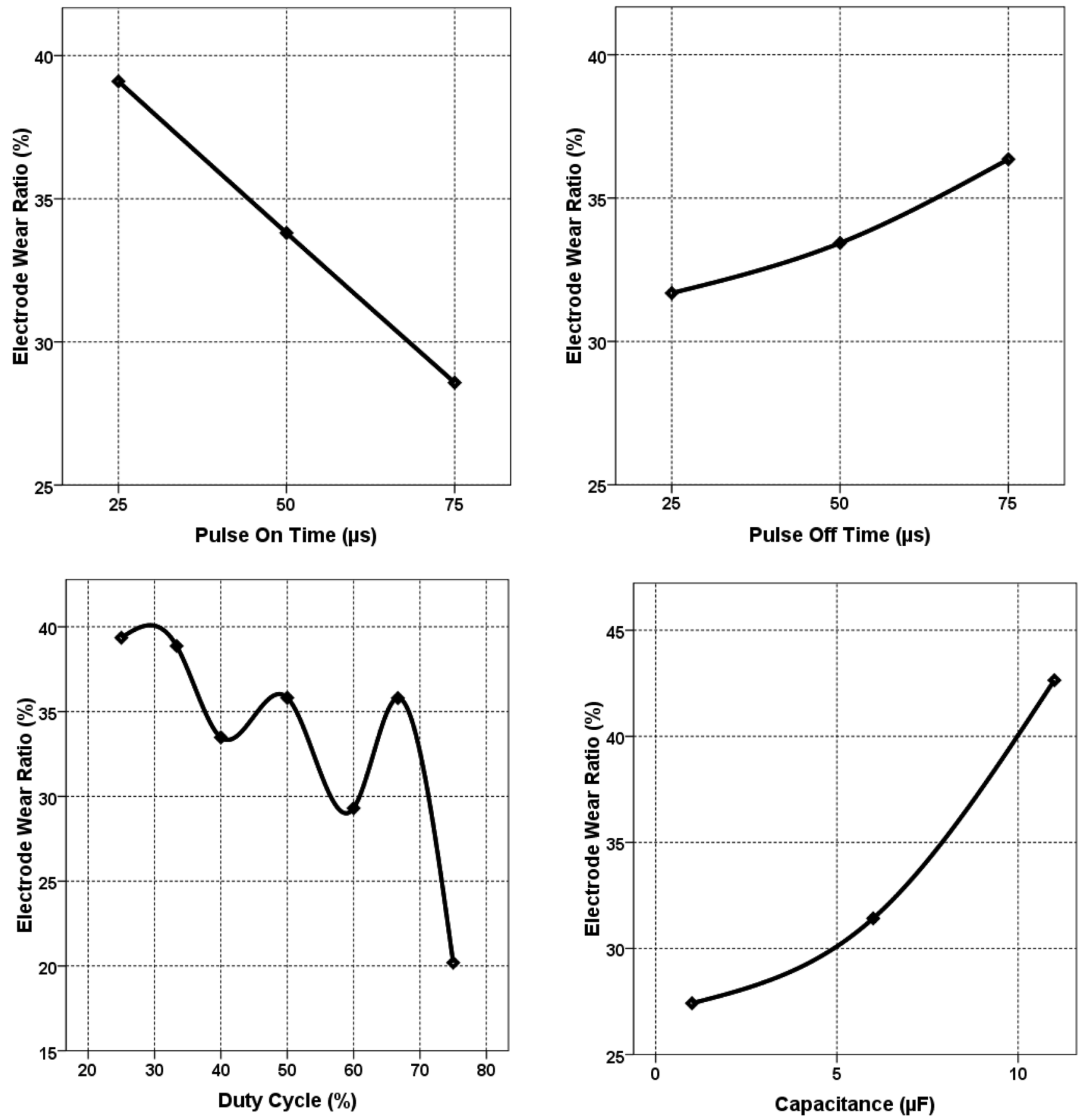

Fig. 4 The effects of electrical parameters, pulse-on/off times, duty cycle and capacitance, on EWR

TOPSIS and GRA methods, whose higher value is desired, were categorized as beneficial criterion. In order to make all these criterions comparable, the normalization decision $N_{i}^{V I K O R}=\frac{\operatorname{Min}\left(X_{i j}\right)}{X_{i j}}$ procedure was applied to performance scores of the first MCDM methods by the following equations. 


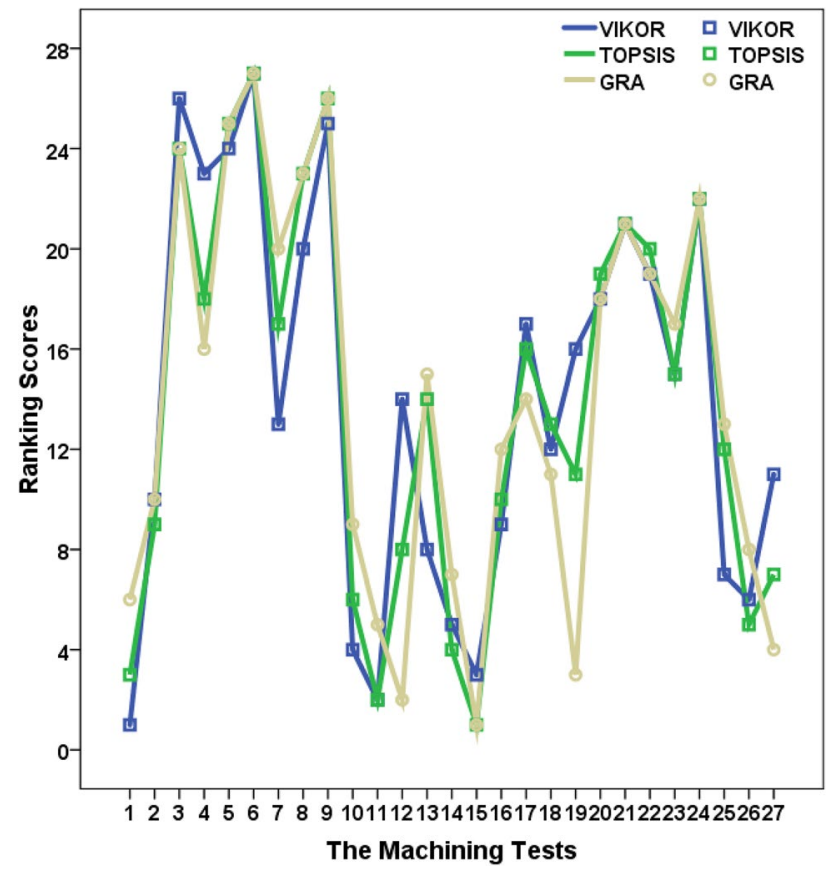

Fig. 5 Results of rank scores depending on different MCDM methods (VIKOR, TOPSIS and GRA)

$N_{i}^{\text {TOPSIS,GRA }}=\frac{X_{i j}}{\operatorname{Max}\left(X_{i j}\right)}$

where $X_{i j}$ is the performance value of ith alternative over jth criterion (performance score). The weightages were assigned equally for the all criterion as $33 \%$. Then, normalized values of each criterion were multiplied by the weightages to find weighted normalized decision matrix. Eventually, in order to obtain performance scores and ranks of the alternatives, all weighted normalized performance values of each alternative were summed by the following equation.

$W S_{i}=\sum_{j=1}^{n} w_{i} x_{i j}$

where $w_{i}$ is the weightage (0.33) of the criterion. In the case of weighted product model, the following equation was employed in order to obtain performance scores and ranks.

$W P_{i}=\prod_{j=1}^{n} X_{i j}^{w_{i}}$

Figure 6 shows ranking scores of WS and WP analyses. It is clear that the consistency of the performances of these two methods are better than first analyses. This graph in Fig. 2 indicates that machining test of number 1 is resulted

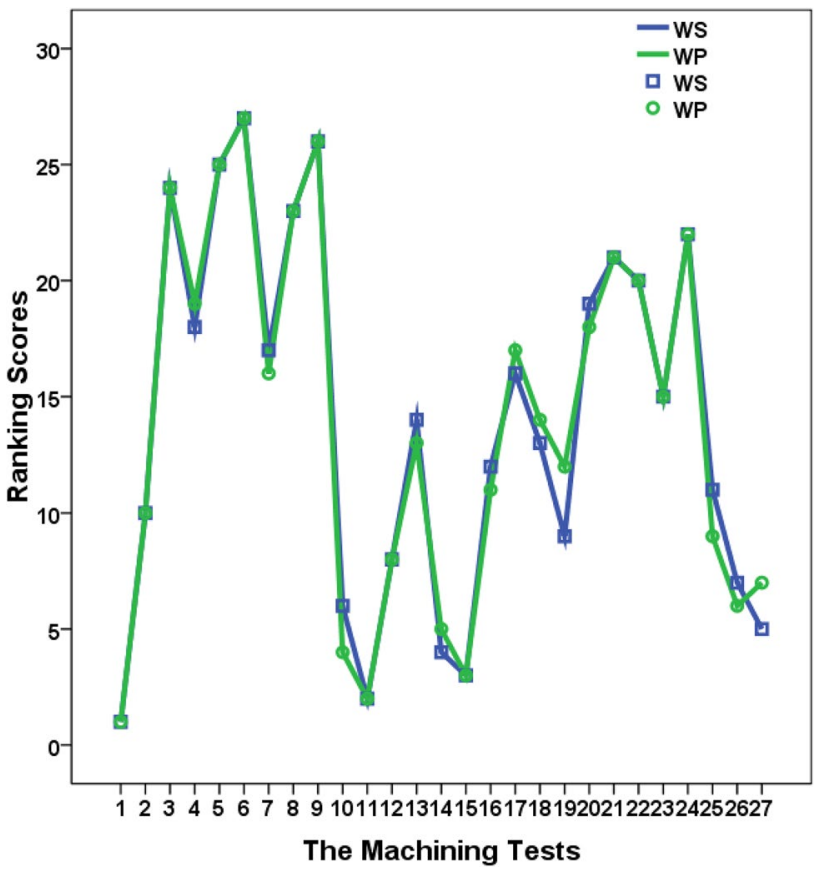

Fig. 6 Results of rank scores depending on weighed sum (WS) and weighted product (WP) models

with the optimal condition as in VIKOR analysis. It has been claimed that in comparison to other methods, VIKOR technique has many advantages in using the MCDM problems especially with opposite criteria [25].

Table 6 shows the experimental interactions of 27 test alternatives. Calculated performance scores by the MCDM methods with respect to these interactions and determined optimal machining conditions with their ranking scores were also shown in this table.

\subsection{Hole formations by meso-scale EDD}

Figure 7 shows sample SEM images of hole formations for capacitances of 1,6 and $11 \mu \mathrm{F}$ at $25 \mu$ sulse-on/off durations. Distinctly, there is a projection of inside diameter of the electrode tool to the workpiece as seen in Fig. 2 c). While the material removal rate was higher in that condition $\left(5.179 \mathrm{~mm}^{3} / \mathrm{min}\right)$, the machining time was lower (47 s) in comparison to others $\left(2.342\right.$ and $3.457 \mathrm{~mm}^{3} / \mathrm{s}-236$ and $122 \mathrm{~s})$. Therefore, the depth of this hole is lower than the others and probably the projection of inside diameter of the electrode tool becomes visible. This results should be considered. Because these unwanted formations in manufacturing could require post machining processes to eliminate it.

\section{SN Applied Sciences}




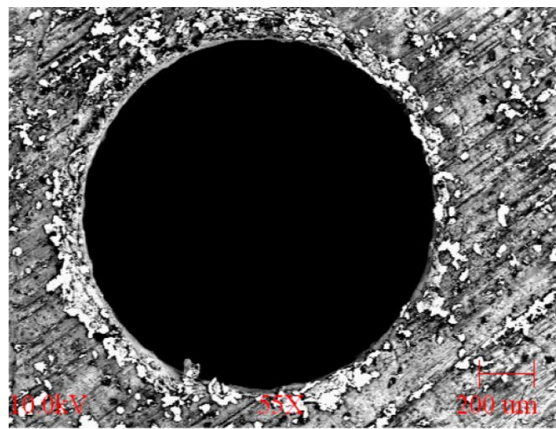

(a)

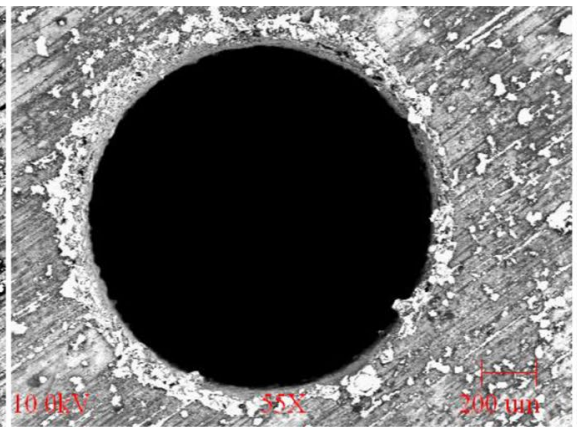

(b)

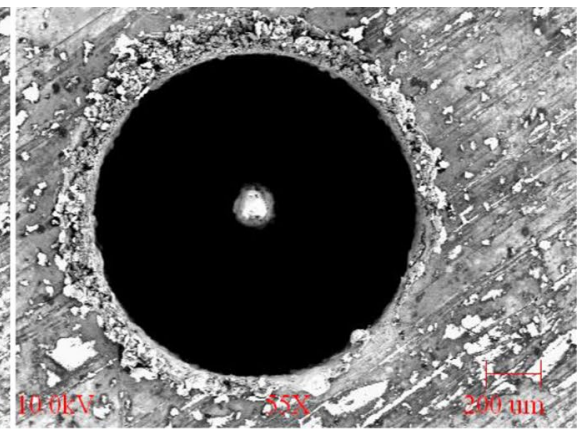

(c)

Fig. 7 Hole formations at $25 \mu$ s pulse-on/off durations for different capacitances a $1 \mu \mathrm{F}, \mathbf{b} 6 \mu \mathrm{F}, \mathbf{c} 11 \mu \mathrm{F}$

\section{Conclusions}

In this study, a machining test (EDD) was done and performance of this test was measured with related to its machining speed (MRR) and tool wear (EWR). Electrical parameters, pulse-on time and duty cycle and interaction of pulse-on/off time have statistically significant effect on MRR for EDD operations. Although, MRR increases with increase of capacitance, its effect on variation of MRR seems to be insignificant. On the other hand, the electrical parameters, pulse-on time, capacitance and duty cycle and the interaction between pulse-on/ off times have significant effect on EWR. For the optimization procedure, MCDM methods (VIKOR, TOPSIS, GRA) were applied to MRR and EWR performance indexes first. VIKOR, one of the multi-criteria decision-making (MCDM) techniques, has proved that the lowest pulse-on/off time values with the $50 \%$ duty cycle and the lowest capacitance value provide the optimum MRR and EWR results for the meso-scale EDD. However, it could not be decided which condition is better in terms of MRR and EWR with the other MCDM methods. Therefore, a second analysis was performed by MS and MP methods. A harmony was achieved by these analyses. Consequently, it has been decided that the test 1 ( $25 \mu$ s pulse-on/off time $50 \%$ duty factor and $1 \mu \mathrm{F}$ capacitance) showed the best performance in terms of machining results of this study. This outcome is also compatible with the result of VIKOR technique. In terms of hole formations, projection of the electrode hole to the workpiece in using of tube electrode should be taken into consideration in EDD operations. For the future aspect, other MCDM methods such as PROMETHEE (preference ranking organization method for enrichment evaluations), ELECTRE (elimination et choice translating reality) and AHP (analytic hierarchy process) could also be experienced and made a comparison for similar machining test conditions. It is suggested that independent and dependent variables could be diversified more for these tests. Results of this study will be beneficial for the manufacturers and researchers working on EDD.

Acknowledgements Author thanks to Professor Kamlakar P. Rajurkar from The University of Nebraska-Lincoln for his contribution to experimental opportunities in the past. Special thanks also to Ala'a Al-okaily, Benny Varghese, Nitin Ingale, Vijay Musunuru and Vineeth Reddy from CNMR (Center for Nontraditional Manufacturing Research) and IMSE 970 (Advanced Manufacturing Processes) course.

\section{Compliance with ethical standards}

Conflict of interest The author declares that he has no conflict of interest.

\section{References}

1. Velasquez M, Hester PT (2013) An analysis of multi-criteria decision making methods. Int J Oper Res 10(2):56-66

2. Behzadian M, Otaghsara SK, Yazdani M, Ignatius J (2012) A state-of the-art survey of TOPSIS applications. Expert Syst Appl 39(17):13051-13069

3. Mardani A, Zavadskas EK, Govindan K, Amat Senin A, Jusoh A (2016) VIKOR technique: a systematic review of the state of the art literature on methodologies and applications. Sustainability $8(1): 37$

4. Ho KH, Newman ST (2003) State of the art electrical discharge machining (EDM). Int J Mach Tools Manuf 43(13):1287-1300

5. Rajurkar KP (1994) Nontraditional manufacturing processes. In: Dorf RC, Kusiak A (eds) Handbook of design, manufacturing and automation. John Wiley \& Sons Inc, New Jersey, pp 211-241

6. El-Hofy HAG (2005) Advanced machining processes: nontraditional and hybrid machining processes. McGraw-Hill, New York

7. Yu Z, Rajurkar KP, Prabhuram PD (2002) Study of contouring micro EDM characteristics. In: Inasaki I (ed) In initiatives of precision engineering at the beginning of a millennium. Springer, Boston, pp 199-203

8. Kuppan P, Rajadurai A, Narayanan S (2008) Influence of EDM process parameters in deep hole drilling of Inconel 718. Int J Adv Manuf Technol 38(1-2):74-84 
9. Chiang KT (2008) Modeling and analysis of the effects of machining parameters on the performance characteristics in the EDM process of $\mathrm{Al}_{2} \mathrm{O}_{3}+\mathrm{TiC}$ mixed ceramic. Int J Adv Manuf Technol 37(5-6):523-533

10. Jahan MP, Wong YS, Rahman M (2009) A study on the fine-finish die-sinking micro-EDM of tungsten carbide using different electrode materials. J Mater Process Technol 209(8):3956-3967

11. Mahardika M, Prihandana GS, Endo T, Tsujimoto T, Matsumoto $\mathrm{N}$, Arifvianto B, Mitsui K (2012) The parameters evaluation and optimization of polycrystalline diamond micro-electrodischarge machining assisted by electrode tool vibration. Int J Adv Manuf Technol 60(9-12):985-993

12. Kachhap S, Singh A, Kumar S (2019) Performance evaluation of different electrode geometries in electric discharge drilling of MMCs. Int J Mech Eng Rob Res 8(4):531-535

13. Yadav S, Verma RK (2019) Multi Objective Optimization Modules in Electrical Discharge Machining processes. Int J Appl Eng Res 14(2):162-168

14. Soota T (2020) Multi-response optimization of machining parameter for Zircaloy by response surface methodology and grey relation analysis. Mater Today: Proc 21:1544-1550

15. Payal H, Maheshwari S, Bharti PS (2019) Parametric optimization of EDM process for Inconel 825 using GRA and PCA approach. J Inf Opt Sci 40(2):291-307

16. Pradhan MK (2018) Optimisation of EDM process for MRR, TWR and radial overcut of D2 steel: a hybrid RSM-GRA and entropy weight-based TOPSIS approach. Int J Ind Syst Eng 29(3):273-302

17. Bhuyan R, Routara B (2016) Optimization the machining parameters by using VIKOR and Entropy Weight method during EDM process of Al-18\% SiCp Metal matrix composite. Decis Sci Lett 5(2):269-282

18. Pattnaik SK, Priyadarshini M, Mahapatra KD, Mishra D, Panda S (2015) Multi objective optimization of EDM process parameters using fuzzy TOPSIS method. In 2015 international conference on innovations in information, embedded and communication systems (ICIIECS) IEEE pp 1-5

19. Guo Y, Zhang G, Wang L, Hu Y (2016) Optimization of parameters for EDM drilling of thermal-barrier-coated nickel superalloys using gray relational analysis method. Int J Adv Manuf Technol 83(9-12):1595-1605

20. Manivannan R, Kumar MP (2017) Multi-attribute decisionmaking of cryogenically cooled micro-EDM drilling process parameters using TOPSIS method. Mater Manuf Processes 32(2):209-215

21. Bhosle RB, Sharma SB (2017) Multi-performance optimization of micro-EDM drilling process of Inconel 600 alloy. Mater Today: Proc 4(2):1988-1997
22. Sapkal SU, Jagtap PS (2018) Optimization of micro EDM drilling process parameters for titanium alloy by rotating electrode. Procedia Manuf 20:119-126

23. Garimella PS (1995) A comprehensive study of wire electrical discharge machining of beryllium-copper alloys. Doctoral dissertation, University of Nebraska-Lincoln

24. Opricovic S (1998) Multi criteria optimization of civil engineering systems. PhD Thesis, Faculty Civ Eng, Belgrade

25. Liao H, Xu Z (2013) A VIKOR-based method for hesitant fuzzy multi-criteria decision making. Fuzzy Optim Decis Making 12(4):373-392

26. Tzeng GH, Huang JJ (2011) Multiple attribute decision making: methods and applications. CRC Press, Berlin

27. Deng JL (1989) Introduction to grey system theory. J Grey Syst $1(1): 1-24$

28. Gholipoor A, Shabgard MR, Mohammadpourfard M (2020) A novel approach to plasma channel radius determination and numerical modeling of electrical discharge machining process. J Braz Soc Mech Sci Eng 42(4):1-10

29. Yan BH, Wang CC, Liu WD, Huang FY (2000) Machining characteristics of Al2O3/6061Al composite using rotary EDM with a disklike electrode. Int J Adv Manuf Technol 16(5):322-333

30. Mohan B, Rajadurai A, Satyanarayana KG (2004) Electric discharge machining of $\mathrm{Al}-\mathrm{SiC}$ metal matrix composites using rotary tube electrode. J Mater Process Technol 153:978-985

31. Chattopadhyay KD, Verma S, Satsangi PS, Sharma PC (2009) Development of empirical model for different process parameters during rotary electrical discharge machining of coppersteel (EN-8) system. J Mater Process Technol 209(3):1454-1465

32. Singh NK, Pandey PM, Singh KK (2016) EDM with an airassisted multi-hole rotating tool. Mater Manuf Processes 31(14):1872-1878

33. Liu P, Zhang L (2015) The extended VIKOR method for multiple criteria decision making problem based on neutrosophic hesitant fuzzy set. arXiv preprint arXiv:1512.0139

34. Mathew M, Sahu S, Upadhyay AK (2017) Effect of normalization techniques in robot selection using weighted aggregated sum product assessment. Int J Innov Res Adv Stud 4:59-63

Publisher's Note Springer Nature remains neutral with regard to jurisdictional claims in published maps and institutional affiliations. 